\title{
Preoperative cardiac evaluation with transthoracic echocardiography before non-cardiac surgery
}

\author{
Chi Young Shim \\ Cardiology Division, Severance Cardiovascular Hospital, Yonsei University College of Medicine, Seoul, Korea
}

Transthoracic echocardiography has become increasingly popular in clinical practice. It is used for the functional evaluation of patients with various cardiovascular diseases. Its use has been extended further in routine screening for cardiovascular health and in preoperative risk assessment before non-cardiac surgery because it is non-invasive, easy to perform, reproducible, and cost-effective. When the results of preoperative echocardiography contain abnormalities, the findings must be interpreted to determine clinical relevance. However, when the results of preoperative echocardiography are apparently normal, many physicians and surgeons readily think that the patient will not have any cardiovascular events in the future, or at least in the perioperative period. In this review, we will cover 1) current guidelines for preoperative echocardiographic assessment, 2) specific cardiac conditions for which the non-cardiac surgery should be delayed, 3) commonly encountered echocardiographic findings before non-cardiac surgeries, 4) application of stress echocardiography, and 5) clinical perspectives of focused transthoracic echocardiography before non-cardiac surgery.

Key Words: Cardiac risk, Echocardiography, Non-cardiac, Surgery.

\section{Introduction}

With an aging population that continues to grow, many patients have cardiovascular risk factors or preexisting cardiovascular disease at the time of non-cardiac surgery. Among various diagnostic imaging modalities for cardiovascular disease, transthoracic echocardiography is one of the most popular methods

Corresponding author: Chi Young Shim, M.D., Ph.D.

Cardiology Division, Severance Cardiovascular Hospital, Yonsei University College of Medicine, 50, Yonsei-ro, Seodaemun-gu, Seoul 03722, Korea

Tel: 82-2-2228-8453, Fax: 82-2-2227-7432

Email: cysprs@yuhs.ac

ORCID: https://orcid.org/0000-0002-6136-0136

Received: July 3, 2017.

Accepted: July 7, 2017.

Korean J Anesthesiol 2017 August 70(4): 390-397

https://doi.org/10.4097/kjae.2017.70.4.390 for preoperative cardiac risk stratification because it can simultaneously evaluate cardiac structures and the function of the heart [1]. Moreover, it is safe, reproducible, cost-effective, and easy to perform. Thus, echocardiography is used widely and its potential applications are increasing with advances in technology. Appropriate knowledge regarding indications for echocardiography in the current guidelines, applications of echocardiography in real clinical practice, and a correct understanding of commonly encountered echocardiographic findings might help to manage patients in the perioperative period, and occasionally rescue patients from acute manifestations of overt cardiovascular diseases, such as decompensated heart failure or cardiogenic shock.

\section{Current Guidelines: When to Perform Preoperative Echocardiography?}

Current guidelines, in fact, do not recommend routine transthoracic echocardiography for the evaluation of cardiac function before non-cardiac surgery. Table 1 summarizes the recommen-

(c) This is an open-access article distributed under the terms of the Creative Commons Attribution Non-Commercial License (http://creativecommons.org/ licenses/by-nc/4.0/), which permits unrestricted non-commercial use, distribution, and reproduction in any medium, provided the original work is properly cited. 
dations for echocardiography before non-cardiac surgery in several guidelines. The British Society of Echocardiography guidelines [2], published in April 2013, comprehensively stated the clinical indications for echocardiography for elective and semiurgent surgery. In this guideline, four indications are specified: (1) documented ischemic heart disease with reduced functional capacity ( $<4$ metabolic equivalents [METs]), (2) unexplained shortness of breath in the absence of clinical signs of heart failure if the electrocardiogram and/or chest X-ray is abnormal, (3) murmur in the presence of cardiac or respiratory symptoms, and (4) murmur in an asymptomatic individual in whom clinical features or other investigations suggest severe structural heart disease. To meet these indications, we need a properly taken history showing any previous ischemic heart disease and an accurate physical examination, including auscultation. In fact, today, primary physicians' and trainees' ability to hear cardiac murmur may be poor, as various imaging modalities have been developed. In real clinical practice in Korea, many hospitals have their own practical standards for performing preoperative echocardiography before non-cardiac surgery. For example, in elderly patients without a completely normal electrocardiogram or chest X-ray, many physicians request preoperative echocardiography. However, repeated assessments versus a previous echocardiogram with no intervening change in clinical status and routine preoperative echocardiography are not indicated.

In the guidelines of the American Society of Echocardiogra- phy [3], there is no clear defined indication for resting echocardiograms. Additionally, the American College of Cardiology/ American Heart Association (ACC/AHA) guidelines published in 2014 defined the indications for echocardiography in a different way [4]. It is recommended that patients with clinically suspected moderate or greater degrees of valvular stenosis or regurgitation undergo preoperative echocardiography if there has been (1) no prior echocardiography within 1 year, or (2) a significant change in clinical status or physical examination since the last evaluation. It is reasonable for patients with dyspnea of unknown origin to undergo preoperative evaluation of left ventricular (LV) function. It is also reasonable for patients with heart failure with worsening dyspnea or other change in clinical status to undergo preoperative evaluation of LV function. Reassessment of LV function in clinically stable patients with previously documented LV dysfunction may be considered if there has been no assessment within 1 year. However, also in these guidelines, routine preoperative evaluation of LV function is not recommended.

Under consideration of appropriate indications of preoperative echocardiography in a few current guidelines, we should apply echocardiography in cost- and time-effective ways in clinical practice. Thus, the next step is to assess individual risk based on the planned, non-cardiac surgical procedures and to exclude active cardiac conditions that are required to undergo evaluation and treatment before non-cardiac surgery.

Table 1. Recommendations for Echocardiography before Non-cardiac Surgery Under Current Guidelines

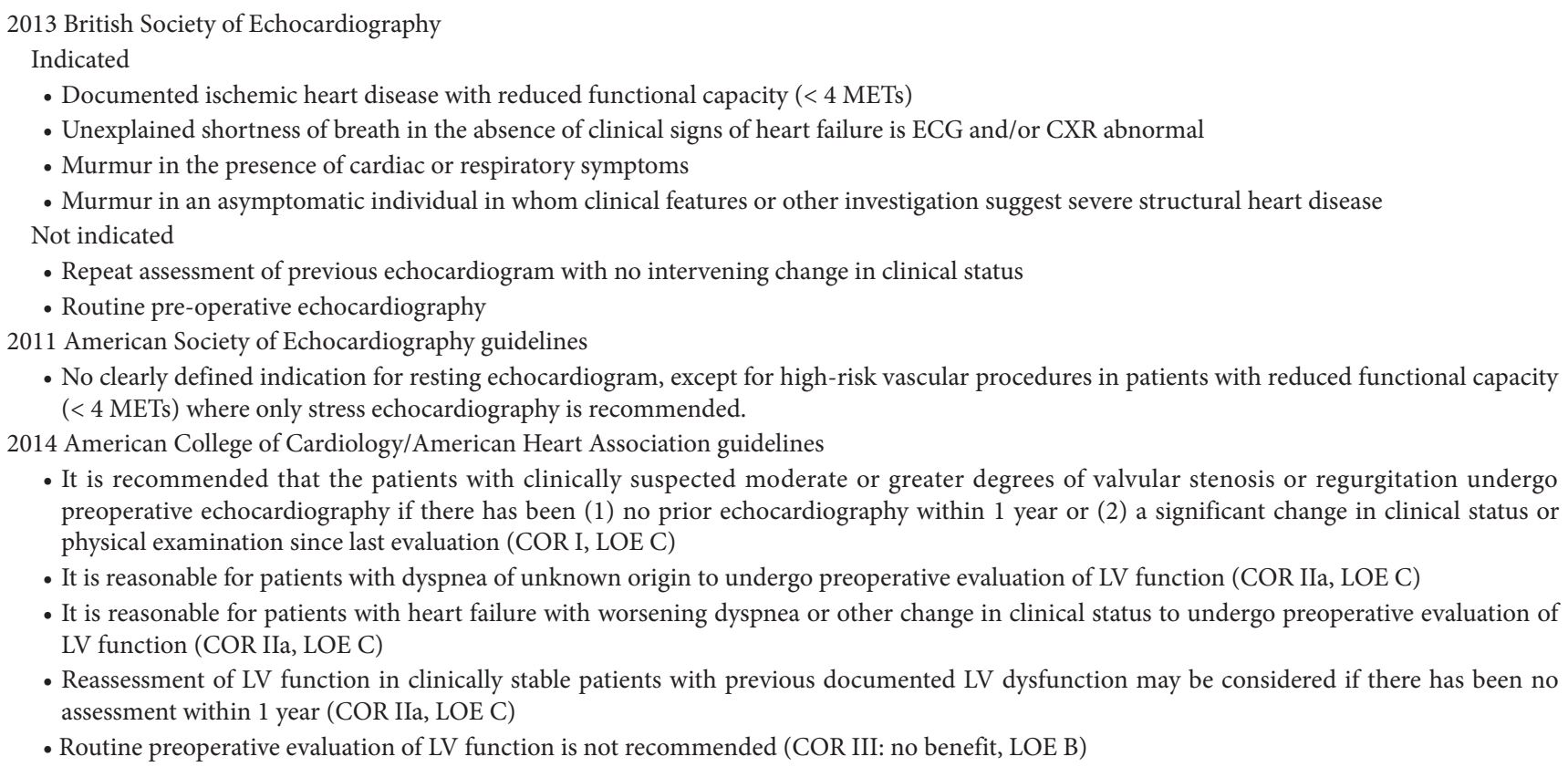

METs: metabolic equivalents, ECG: electrocardiogram, CXR: chest X-ray, COR: class of recommendation, LOE: level of evidence, LV: left ventricular. 


\section{Factors to Be Considered When Assessing Cardiac Risk}

Cardiac complications after non-cardiac surgery depend on patient-related risk factors and on the type of surgery $[5,6]$. Thus, before considering a preoperative cardiac evaluation, including echocardiography, individual cardiac risk related to the surgical procedures should be assessed [7]. Three factors that must be considered are listed in Table 2 [8].

Patient-related factors include age, presence of chronic disease, functional status, medical history, and the presence of implantable devices or prior surgical history. Regarding functional status, a history of daily activities for individuals must be taken. If the patient can climb a flight of stairs or walk up a hill, his or her metabolic equivalent is $>4$ METs. If the patient can participate in strenuous sports like swimming, singles tennis, football, baseball, or skiing, his or her metabolic equivalent is $>10$ METs. The inability to walk $\geq 2$ blocks on level ground or run a short distance ( $<4$ METs) indicates poor functional capacity and is

Table 2. Factors to Be Considered When Assessing Cardiac Risk

Patient-related factors
Age
Chronic disease (e.g., coronary artery disease, diabetes mellitus,
or hypertension)
Functional status
Medical history
Implantable devices
Previous surgeries
Surgery-related factors
Type of surgery (e.g., vascular, endoscopic, or abdominal)
Urgency status (e.g., emergency, urgent, or elective)
Surgical duration, possibility of blood loss and fluid shifts
Test-related factors
Sensitivity and specificity of a test
Effect on management

associated with an increased incidence of post-operative cardiac events $[9,10]$. Information about functional capacity is important in deciding whether to do additional stress testing. It is reasonable for patients who are at an elevated risk for non-cardiac surgery and have poor functional capacity ( $<4$ METs) to undergo non-invasive pharmacological stress testing (dobutamine stress echocardiography or pharmacological-stress nuclear imaging) under the ACC/AHA guidelines. Advanced age adversely affects the rates of cardiac and non-cardiac complications and mortality, as well as length of stay. Perioperative mortality risk was in the intermediate range $(2.6 \%$ in patients $>80$ years vs. $0.3 \%$ in patients $50-59$ years of age, $\mathrm{P}=0.02$ ) according to the results from a prospective cohort analysis by Polanczyk et al. [11] in Brigham \& Women's Hospital. However, there is no reported evidence that routine preoperative evaluation using echocardiography can improve perioperative outcomes in elderly patients.

Surgery-related factors include the type of surgery, urgency status, surgical duration, and the possibility of blood loss and fluid shifts. Table 3 shows the cardiac risk stratification based on surgical procedures. Vascular procedures, such as aortic and other major vascular surgery are at a high cardiac risk because the reported cardiac risk is often $>5 \%[12,13]$. However, endoscopic procedures, superficial procedures, cataract surgery, breast surgery, and common ambulatory surgeries are at a low cardiac risk $(<1 \%)[14,15]$. Most elective non-cardiac surgeries are at an intermediate cardiac risk (1-5\%).

Finally, before considering specific tests, we need to consider test-related factors. Patients, their family members, and even surgeons usually believe that the sensitivity and specificity of a specific test for cardiac evaluation are $100 \%$. Although this is not true, if the results from some specific cardiac test are normal, they simply consider the patient's heart condition to be normal. For example, while there is no regional wall motion abnormality and normal LV ejection fraction in resting transthoracic echo-

Table 3. Cardiac Risk Stratification for Non-cardiac Surgical Procedures

\begin{tabular}{ll}
\hline \multicolumn{1}{c}{ Risk $^{*}$ stratification } & \multicolumn{1}{c}{ Procedure examples } \\
\hline High (reported cardiac risk often $>5 \%)$ & Aortic and other major vascular surgery \\
& Peripheral vascular surgery \\
Intermediate (reported cardiac risk generally 1-5\%) & Intraperitoneal and intrathoracic surgery \\
& Carotid endarterectomy \\
& Head and neck surgery \\
Low (reported cardiac risk generally $<1 \%)$ & Orthopedic surgery \\
& Prostate surgery \\
& Endoscopic procedures \\
& Superficial procedure \\
& Cataract surgery \\
& Breast surgery \\
& Ambulatory surgery
\end{tabular}

${ }^{*}$ Risk of myocardial infarction and cardiac death within 30 days after surgery. 
cardiography, there are still possibilities for acute coronary syndrome, such as unstable angina or non-ST elevation myocardial infarction on the LV. Thus, we need to understand the potential limitations of non-invasive tests, know the sensitivity and specificity to identify specific cardiovascular diseases, and consider the effects on management.

\section{Active Cardiac Conditions to Delay Non-cardiac Surgery}

Although the risk stratification of patients before non-cardiac surgery in stable condition is valuable in preventing cardiac events in the perioperative period, the most important purpose of preoperative screening is excluding active cardiac conditions. Table 4 displays four categories of active cardiac conditions [16]. If the patient has one of these conditions, the elective noncardiac surgery should be delayed until the patient is out of the condition and has become stable.

The four conditions are unstable coronary syndromes, decompensated heart failure, significant arrhythmia, and severe valvular disease. To define the presence of severe valvular disease, quantitative measurements of valvular hemodynamics by echocardiography are essential. Additionally, to recognize the three conditions except severe valvular disease, echocardiography is not necessary but valuable in defining the underlying cause(s), assessing the severity of each condition, evaluating the treatment response, and predicting the prognosis.

Today, as the aged population grows, the incidence of severe aortic stenosis due to degenerative causes will increase. Severe aortic stenosis is defined according to an integrative approach taking into account valve area $\left(<1.0 \mathrm{~cm}^{2}\right.$ or $0.6 \mathrm{~cm}^{2} / \mathrm{m}^{2}$ body surface area, except in obese patients), and flow-dependent indices (maximum jet velocity of $4 \mathrm{~m} / \mathrm{s}$ and mean aortic pressure gradient of $\geq 40 \mathrm{mmHg}$ ) [17]. It is often detected during preoperative cardiac evaluations. Among the various valvular diseases, severe stenotic disease at the aortic valve may affect the clinical course seriously during general anesthesia and/or after noncardiac surgery [18]. Severe aortic stenosis is a well-established risk factor for perioperative mortality and myocardial infarction [7]. In cases of urgent non-cardiac surgery in patients with severe aortic stenosis, such procedures should be performed under more invasive hemodynamic monitoring, avoiding rapid changes in volume status and heart rhythm as much as possible. In the case of elective non-cardiac surgery, the presence of symptoms is essential for decision-making [19]. In symptomatic patients, aortic valve replacement should be considered before an elective surgery [19]. In patients at high risk or contraindicated for aortic valve replacement, transcatheter aortic valve implantation may be a reasonable therapeutic option before surgery [20,21]. In asymptomatic patients, non-cardiac surgery of low-to-intermediate risk can be performed. If possible, the absence of symptoms should be confirmed by exercise testing. If high-risk surgery is planned, further clinical assessment is needed to assess the risk of aortic valve replacement [7].

In terms of mitral stenosis, asymptomatic mitral stenosis is not absolutely contraindicated but symptomatic mitral stenosis should be treated by percutaneous mitral valvuloplasty or mitral valve replacement [7]. However, even in asymptomatic patients with significant mitral stenosis, systolic pulmonary artery pressure over $50 \mathrm{mmHg}$ is a risk factor for clinical deterioration in

Table 4. Active Cardiac Conditions That Are Contraindication for Non-cardiac Surgery

\begin{tabular}{ll}
\hline \multicolumn{1}{c}{ Conditions } & \multicolumn{1}{c}{ Examples } \\
\hline Unstable coronary syndromes & Unstable or severe angina (CCS class III or IV)* \\
& Recent myocardial infarction ${ }^{\dagger}$ \\
$\begin{array}{l}\text { Decompensated heart failure (NYHA functional class IV; } \\
\text { worsening or new-onset heart failure) }\end{array}$ & \\
Significant arrhythmias & High-grade atrioventricular block \\
& Mobitz II atrioventricular block \\
& Third-degree atrioventricular heart block \\
& Supraventricular arrhythmias (including atrial fibrillation) with uncontrolled \\
& ventricular rate (HR $>100$ beats/min at rest) \\
& Symptomatic bradycardia \\
& Newly recognized ventricular tachycardia \\
& Severe aortic stenosis (mean pressure gradient $>40$ mmHg, aortic valve area $<1.0 \mathrm{~cm}^{2}$, \\
Severe valvular disease & or symptomatic) \\
& Symptomatic mitral stenosis (progressive dyspnea on exertion, exertional presyncope, \\
& or heart failure)
\end{tabular}

(Adapted from Douglas et al. J Am Soc Echocardiogr 2011;24:229-67). CCS: Canadian Cardiovascular Society, NYHA: New York Heart Association, HR: heart rate. *May include "stable" angina in patients who are unusually sedentary. ${ }^{\dagger}$ The American College of Cardiology National Database Library defines recent myocardial infarction as $>7$ days but $\leq 1$ month (within 30 days). 
perioperative periods. Additionally, combined atrial fibrillation or tachycardia may cause serious clinical deterioration. Thus, use of $\beta$-blockers or calcium channel blockers to keep a sufficient diastolic filling time may be helpful in preventing perioperative decompensated heart failure.

\section{Cardiac Risk Stratification for Non-cardiac Surgical Procedures}

If patients do not have an active cardiac condition, those at risk for major adverse cardiac events before non-cardiac surgery still need to be identified. To stratify cardiac risk, the ACC/AHA have created a classification of non-cardiac surgical procedures, as shown in Table 3 [3]. In fact, procedures in the intermediate category cover a wide range of surgical procedures and carry a $1-5 \%$ risk of adverse cardiac events. Thus, clinical risk factors such as a prior history of heart disease, compensated or prior heart failure, cerebrovascular disease, diabetes mellitus, and renal insufficiency should be dealt with cautiously if the procedures are in the intermediate risk categories. If a patient has three or more risk factors, a comprehensive cardiovascular evaluation should be considered before non-cardiac surgery. However, if there are one or two clinical risk factors, a preoperative cardiac evaluation is recommended at the physician's discretion.

\section{Interpretation of Common Echocardiographic Findings}

Table 5 lists commonly encountered findings in preoperative echocardiography and their clinical interpretation. These findings are somewhat helpful for risk stratification for future cardiovascular events. Although many of these findings are only subclinical abnormalities or incidental findings, each has potential clinical implications. Additionally, if there are some discrepancies between individual's symptoms and echocardiographic findings, clinical correlations by cardiologists or experienced anesthesiologists are important. For example, if a patient complains of exertional dyspnea but there are no remarkable findings on resting echocardiography, further functional assess-

Table 5. Frequently Encountered Findings in Preoperative Echocardiography

\begin{tabular}{|c|c|}
\hline Echocardiographic findings & Interpretation \\
\hline Left atrial enlargement & $\begin{array}{l}\text { Degree of left atrial enlargement reflects chronic LV diastolic dysfunction. Patients with left atrial enlargement } \\
\text { have an increased risk of atrial fibrillation in postoperative periods. }\end{array}$ \\
\hline Diastolic dysfunction & $\begin{array}{l}\text { Patients with advanced diastolic dysfunction are prone to develop heart failure in volume overloaded } \\
\text { condition ad tachycardia status. }\end{array}$ \\
\hline LV hypertrophy & $\begin{array}{l}\text { LV hypertrophy itself is not usually problematic, but combined diastolic dysfunction is important (above). } \\
\text { However, it should be differentiated from hypertrophic cardiomyopathy or infiltrative myocardial disease. }\end{array}$ \\
\hline Regional wall motion abnormalities & $\begin{array}{l}\text { Common causes of regional wall motion abnormalities are ischemic heart disease. Myocardial disease, } \\
\text { secondary to left bundle branch block, or stress cardiomyopathy should be differentiated from ischemic } \\
\text { heart disease. }\end{array}$ \\
\hline Systolic dysfunction & $\begin{array}{l}\text { LV ejection fraction is a representative indicator for LV systolic function. If a patient has systolic dysfunction, } \\
\text { check E/e' ratio, reflecting LV filling pressure, and right ventricular systolic pressure for evaluation of heart } \\
\text { failure status and possibility for the development of decompensated heart failure. }\end{array}$ \\
\hline Valvular regurgitation & $\begin{array}{l}\text { Intraoperative and postoperative hemodynamic monitoring is reasonable in patients with asymptomatic } \\
\text { severe mitral regurgitation and in patients with asymptomatic severe aortic regurgitation and a normal } \\
\mathrm{LV} \text { ejection fraction. }\end{array}$ \\
\hline Valvular stenosis & $\begin{array}{l}\text { For patients who meet standard indications for valvular intervention on the basis of symptoms and severity } \\
\text { of stenosis, valvular intervention before non-cardiac surgery is effective in reducing preoperative risk. } \\
\text { Intraoperative and postoperative hemodynamic monitoring is reasonable in patients with asymptomatic } \\
\text { severe aortic stenosis and in asymptomatic patients with severe mitral stenosis if valve morphology is not } \\
\text { favorable for percutaneous mitral balloon commissurotomy. }\end{array}$ \\
\hline Prosthetic valve & $\begin{array}{l}\text { All patients with prosthetic valves should receive antibiotic prophylaxis before non-cardiac surgery. } \\
\text { Patients with mechanical valves pose a special problem regarding anticoagulation. }\end{array}$ \\
\hline Pericardial effusion & $\begin{array}{l}\text { The amount of pericardial effusion and presence of hemodynamic significance, such as constrictive physiology } \\
\text { or tamponade physiology, are carefully interpreted. Avoiding intracardiac volume depletion and } \\
\text { monitoring blood pressure and heart rate are crucial in patients with significant amounts of pericardial } \\
\text { effusion. }\end{array}$ \\
\hline Pulmonary hypertension & $\begin{array}{l}\text { The presence of moderate-to-severe pulmonary hypertension is commonly combined with left heart } \\
\text { disease. Elevation of estimated pulmonary venous pressure can be a sign of decompensated heart failure. } \\
\text { Pulmonary arterial hypertension due to congenital heart disease or idiopathic origin is at a high risk for } \\
\text { non-cardiac surgery. If a patient has right ventricular systolic pressure over } 50 \mathrm{mmHg} \text {, consider invasive } \\
\text { hemodynamic monitoring during and after non-cardiac surgery. }\end{array}$ \\
\hline
\end{tabular}

LV: left ventricle. 
ments using exercise tests should be considered.

\section{When to Perform Stress Echocardiography Before Non-cardiac Surgery}

There are various types of stress testing for evaluating ischemic heart disease. Stress echocardiography using exercise or pharmacological (dobutamine, dipyridamole) stress has been used widely in preoperative cardiac risk evaluations. The test combines information on LV function at rest, heart valve abnormalities, and the presence and extent of stress-induced ischemia $[7,22]$. However, before non-cardiac surgery, stress testing is mostly focused on the diagnosis and risk stratification of coronary artery disease. For patients with elevated risk and poor $(<4$ METs) or unknown functional capacity, exercise or non-invasive pharmacological stress testing for myocardial ischemia is recommended if it will change management. However, routine screening with non-invasive stress testing is not useful for patients at low risk for non-cardiac surgery $[3,15]$.

When stress testing is required to detect myocardial ischemia, which stress test should be used? Generally, most ambulatory patients commonly undergo a treadmill stress test, but exercise echocardiography or nuclear imaging is preferred in patients with abnormal resting electrocardiograms, including LV hypertrophy strain pattern or digitalization effects. In addition, patients unable to exercise or patients with left bundle branch block on an electrocardiogram should preferably undergo dobutamine stress echocardiography or nuclear imaging. Thus, the stress mode should be selected depending on the clinical situation to reach an appropriate diagnosis.

A previous meta-analytic comparison of preoperative stress echocardiography and nuclear scintigraphy imaging by Beattie et al. [23] demonstrated that stress echocardiography had better negative predictive characteristics than thallium imaging in a comparison of 3,373 subjects who underwent stress echocardiography in 25 studies with 6,827 subjects who underwent thallium imaging in 50 studies. Thus, the conclusion was that stress echocardiography was superior to thallium imaging in predicting postoperative cardiac events. Generally, stress echocardiography has a high negative predictive value and a negative test is associated with a very low incidence of cardiac events in patients undergoing surgery. In another meta-analysis of six non-invasive tests by Kertai et al. [24], dobutamine stress echocardiography showed a positive trend towards better diagnostic performance versus the other tests, including ambulatory electrocardiogram, exercise electrocardiogram, radionuclide ventriculography, dipyridamole stress echocardiography, and myocardial perfusion scintigraphy.

\section{Focused Echocardiography for Preoperative Cardiac Evaluation}

Recently, interest in focused echocardiography has increased for evaluating cardiac status effectively for a specific goal. Particularly, increased availability, portability, and imaging capabilities of current transthoracic echocardiography equipment has enabled treating physicians to perform their own 'focused echocardiography' as an aid to an initial clinical assessment at the point of care, when required. For example, aortic stenosis, common in the hip fracture population, and pulmonary hypertension are significant risk factors for mortality, but diagnosis is unreliable without echocardiography. Thus, focused echocardiography may provide a more accurate assessment than a physical examination alone. Attractively, it is usually performed at the patient's bedside as a point of care.

A prospective observational study by Canty et al. [25] discussed focused transthoracic echocardiography in 99 patients who had suspected cardiac disease or were $\geq 65$ years old. Focused echocardiography included only a few important points of LV or right ventricular failure, valve stenosis, or regurgitation of at least moderate severity, pericardial effusion greater than $0.5 \mathrm{~cm}$, and estimated pulmonary arterial systolic pressure $>60 \mathrm{mmHg}$. In that study, cardiac disease was identified by echocardiography in $64 \%$ of patients, which led to a step-up of treatment in 36\% (4\% delayed for cardiology referral, $2 \%$ altered surgery, $4 \%$ intensive care, and $25 \%$ intra-operative hemodynamic management changes). Absence of cardiac disease in $36 \%$ resulted in a step-down of treatment in $8 \%$ (3\% no referral, $1 \%$ intensive care, and $4 \%$ hemodynamic treatment). Although further studies are warranted, focused echocardiography is a valuable method for preoperative cardiac risk stratification.

\section{Conclusions}

In the current guidelines, routine preoperative cardiac evaluation by echocardiography is not recommended for everyone, given the considerations of cost-effectiveness and time delay for non-cardiac surgery. Preoperative echocardiography has become more popular in clinical practice because it can provide clinically useful information about a patient's cardiac risk. Thus, understanding the commonly encountered echocardiographic findings and clinical interpretations are helpful for managing patients in the perioperative period. 


\section{References}

1. Ryu T, Song SY. Perioperative management of left ventricular diastolic dysfunction and heart failure: an anesthesiologist's perspective. Korean J Anesthesiol 2017; 70: 3-12.

2. British Society of Echocardiography. Clinical indications for echocardiography. [Last accessed on April 2013]. Available from https://www. bsecho.org/indications-for-echocardiography/

3. American College of Cardiology Foundation Appropriate Use Criteria Task Force, American Society of Echocardiography, American Heart Association, American Society of Nuclear Cardiology, Heart Failure Society of America, Heart Rhythm Society, et al. ACCF/ASE/AHA/ ASNC/HFSA/HRS/SCAI/SCCM/SCCT/SCMR 2011 Appropriate use criteria for echocardiography. A report of the American College of Cardiology Foundation Appropriate Use Criteria Task Force, American Society of Echocardiography, American Heart Association, American Society of Nuclear Cardiology, Heart Failure Society of America, Heart Rhythm Society, Society for Cardiovascular Angiography and Interventions, Society of Critical Care Medicine, Society of Cardiovascular Computed Tomography, Society for Cardiovascular Magnetic Resonance American College of Chest Physicians. J Am Soc Echocardiogr 2011; 24: 229-67.

4. Fleisher LA, Fleischmann KE, Auerbach AD, Barnason SA, Beckman JA, Bozkurt B, et al. 2014 ACC/AHA guideline on perioperative cardiovascular evaluation and management of patients undergoing noncardiac surgery: executive summary: a report of the American College of Cardiology/American Heart Association Task Force on Practice Guidelines. Circulation 2014; 130: 2215-45.

5. Wirthlin DJ, Cambria RP. Surgery-specific considerations in the cardiac patient undergoing noncardiac surgery. Prog Cardiovasc Dis 1998; 40: $453-68$.

6. Goldman L, Caldera DL, Nussbaum SR, Southwick FS, Krogstad D, Murray B, et al. Multifactorial index of cardiac risk in noncardiac surgical procedures. N Engl J Med 1977; 297: 845-50.

7. Fleisher LA. Cardiac risk stratification for noncardiac surgery: update from the American College of Cardiology/American Heart Association 2007 guidelines. Cleve Clin J Med 2009; 76 Suppl 4: S9-15.

8. Kristensen SD, Knuuti J, Saraste A, Anker S, Bøtker HE, Hert SD, et al. 2014 ESC/ESA Guidelines on non-cardiac surgery: cardiovascular assessment and management: The Joint Task Force on non-cardiac surgery: cardiovascular assessment and management of the European Society of Cardiology (ESC) and the European Society of Anaesthesiology (ESA). Eur Heart J 2014; 35: 2383-431.

9. Wiklund RA, Stein HD, Rosenbaum SH. Activities of daily living and cardiovascular complications following elective, noncardiac surgery. Yale J Biol Med 2001; 74: 75-87.

10. Morris CK, Ueshima K, Kawaguchi T, Hideg A, Froelicher VF. The prognostic value of exercise capacity: a review of the literature. Am Heart J 1991; 122: 1423-31.

11. Polanczyk CA, Marcantonio E, Goldman L, Rohde LE, Orav J, Mangione CM, et al. Impact of age on perioperative complications and length of stay in patients undergoing noncardiac surgery. Ann Intern Med 2001; 134: 637-43.

12. Stather PW, Sidloff D, Dattani N, Choke E, Bown MJ, Sayers RD. Systematic review and meta-analysis of the early and late outcomes of open and endovascular repair of abdominal aortic aneurysm. Br J Surg 2013; 100: 863-72.

13. Paravastu SC1, Jayarajasingam R, Cottam R, Palfreyman SJ, Michaels JA, Thomas SM. Endovascular repair of abdominal aortic aneurysm. Cochrane Database Syst Rev 2014; (1): CD004178.

14. Wang CL, Qu G, Xu HW. The short- and long-term outcomes of laparoscopic versus open surgery for colorectal cancer: a meta-analysis. Int J Colorectal Dis 2014; 29: 309-20.

15. Rosenmüller MH, Thorén Örnberg M, Myrnäs T, Lundberg O, Nilsson E, Haapamäki MM. Expertise-based randomized clinical trial of laparoscopic versus small-incision open cholecystectomy. Br J Surg 2013; 100: 886-94.

16. American College of Cardiology Foundation Appropriate Use Criteria Task Force, American Society of Echocardiography, American Heart Association, American Society of Nuclear Cardiology, Heart Failure Society of America, Heart Rhythm Society, et al. ACCF/ASE/AHA/ ASNC/HFSA/HRS/SCAI/SCCM/SCCT/SCMR 2011 Appropriate Use Criteria for Echocardiography. A Report of the American College of Cardiology Foundation Appropriate Use Criteria Task Force, American Society of Echocardiography, American Heart Association, American Society of Nuclear Cardiology, Heart Failure Society of America, Heart Rhythm Society, Society for Cardiovascular Angiography and Interventions, Society of Critical Care Medicine, Society of Cardiovascular Computed Tomography, Society for Cardiovascular Magnetic Resonance American College of Chest Physicians. J Am Soc Echocardiogr 2011; 24: 229-67.

17. Nishimura RA, Otto CM, Bonow RO, Carabello BA, Erwin JP 3rd, Guyton RA, et al. 2014 AHA/ACC guideline for the management of patients with valvular heart disease: a report of the American College of Cardiology/American Heart Association Task Force on Practice Guidelines. J Am Coll Cardiol 2014; 63: e57-185.

18. Agarwal S, Rajamanickam A, Bajaj NS, Griffin BP, Catacutan T, Svensson LG, et al. Impact of aortic stenosis on postoperative outcomes after noncardiac surgeries. Circ Cardiovasc Qual Outcomes 2013; 6: 193-200.

19. Joint Task Force on the Management of Valvular Heart Disease of the European Society of Cardiology (ESC), European Association for Cardio-Thoracic Surgery (EACTS), Vahanian A, Alfieri O, Andreotti F, Antunes MJ, et al. Guidelines on the management of valvular heart disease (version 2012). Eur Heart J 2012; 33: 2451-96.

20. Leon MB, Smith CR, Mack M, Miller DC, Moses JW, Svensson LG, et al. Transcatheter aortic-valve implantation for aortic stenosis in patients who cannot undergo surgery. N Engl J Med 2010; 363: 1597-607. 
21. Smith CR, Leon MB, Mack MJ, Miller DC, Moses JW, Svensson LG, et al. Transcatheter versus surgical aortic-valve replacement in highrisk patients. N Engl J Med 2011; 364: 2187-98.

22. Sicari R, Nihoyannopoulos P, Evangelista A, Kasprzak J, Lancellotti P, Poldermans D, et al. Stress Echocardiography Expert Consensus Statement--Executive Summary: European Association of Echocardiography (EAE) (a registered branch of the ESC). Eur Heart J 2009; 30: 278-89.

23. Beattie WS, Abdelnaem E, Wijeysundera DN, Buckley DN. A meta-analytic comparison of preoperative stress echocardiography and nuclear scintigraphy imaging. Anesth Analg 2006; 102: 8-16.

24. Kertai MD, Boersma E, Bax JJ, Heijenbrok-Kal MH, Hunink MG, L'talien GJ, et al. A meta-analysis comparing the prognostic accuracy of six diagnostic tests for predicting perioperative cardiac risk in patients undergoing major vascular surgery. Heart 2003; 89: $1327-34$.

25. Canty DJ, Royse CF, Kilpatrick D, Williams DL, Royse AG. The impact of pre-operative focused transthoracic echocardiography in emergency non-cardiac surgery patients with known or risk of cardiac disease. Anaesthesia 2012; 67: 714-20. 